\title{
ACUTE CEREBRAL INFARCTION IN WATERSHED DISTRIBUTION IN A PATIENT WITH HYPEREOSINOPHILIC SYNDROME
}

\author{
Guilherme Fleury Perini', Carolina Kassab ${ }^{7}$, Claudia Bley', Paulo Hélio Monzillo², \\ Rodrigo Barbosa Thomaz ${ }^{2}$, Nelson Hamerschlak'
}

Hypereosinophilic syndrome (HES) is a rare myeloid disease characterized by the presence of persistent eosinophilia (for over 6 months) greater than $1500 / \mathrm{mm}^{3}$, associated with organ involvement and dysfunction ${ }^{1,2}$. It is often referred to as idiopathic. The disease affects predominantly males ${ }^{2,3}$, and is extremely rare in children ${ }^{3}$. Clinical manifestations are markedly heterogeneous, since any organ can be vulnerable to eosinophilia-associated tissue damage. However, the major tissue targets are the heart, the central nervous system, the skin and the upper and lower respiratory tract ${ }^{3}$. Eosinophilic patients can remain asymptomatic for decades, and the feature that defines the syndrome is organ involvement ${ }^{2}$.

Diagnosis of HES requires exclusion of all other causes of peripheral eosinophilia and failure of detection of the underlying genetic defect by cytogenetic and/or molecular screening'. Therefore, it is reasonable to perform a complete workup to exclude common causes of eosinophilia, such as parasitic infections, atopy/allergic disorders, malignancy and collagen vascular diseases ${ }^{4}$. The presence of a known cytogenetic defect (such as FIPIL1-PDGRFa, PDGRFb and FGFR1 rearrangements) distinguishes the group of diseases as myeloid neoplasms associated with eosinophilia ${ }^{5}$.

Neurological impairment occurs in up to $65 \%$ of the patients with hypereosinophilic syndrome ${ }^{6}$. Neurological manifestations are often observed in HES, and comprise three types. The first type has a cardiac origin, with thromboemboli causing multiple embolic strokes. The second type comprises one distinct encephalopathy and the third type comprises peripheral neuropathies, which occur in half of the HES patients ${ }^{6}$.

The purpose of this study is to describe the case of a patient with hypereosinophilic syndrome that, despite control of eosinophil count, suffered from neurological impairment.

\section{CASE}

A 63-year-old patient presented to our Service with a twoyear history of sustained eosinophilia. There were no lung and cardiac findings as seen by echocardiography, thorax computed tomography or function tests. She was treated with low corticoesteroid therapy (with prednisone) and used imatinib for two months without response. Her eosinophil count was $40,000 / \mathrm{ml}$ approximately. Her past medical history showed also a relevant deep venous thrombosis in left lower extremity, for which the patient was prescribed with clopidogrel, $75 \mathrm{mg}$ daily, since difficult control was obtained on oral anti-coagulation.

A diagnostic workup excluded usual etiologies for eosinophilia, such as infections, allergy, or other clonal hematopoietic malignancies. The screen for platelet-derived growth factor receptor alpha, PDGFR-a, the protein JAK-2 and the BCR-ABL fusion gene mutations were negative.

One month later, the patient presented with a sudden onset of headache, ataxia and loss of strength and coordination in lower extremities. She demonstrated also spatial disorientation, mental confusion and neglect of her left side. At that time, she had a leukocyte count of $161,800 / \mathrm{ml}$, with 118,400 eosinophils/ $\mathrm{ml}, 12,880$ neutrophils $/ \mathrm{ml}$, and no blasts in peripheral blood. Brain magnetic resonance imaging (MRI) showed extensive bilateral and diffuse watershed ischemia (Figure).

There was no history of hypotensive episode, nor any other end-organ injury that could result from hyperviscosity. An extensive investigation for an embolic source of the infarcts was performed. Transtoracic and transesophagic echocardiogram showed no intracardiac thrombus, but a patent foramen ovale was found with no flow inversion. Left ventricular ejection fraction was preserved, despite myocardial infiltration. Transcranial Doppler study was performed with no embolic activity during more than one hour of measuring, and there was no evidence of arterial stenosis in any segment of intracranial vessels.

The patient was started on $60 \mathrm{mg}$ enoxaparin subcutaneous-

\section{INFARTO CEREBRAL AGUDO COM DISTRIBUIÇÃO MARGINAL EM PACIENTE COM SÍNDROME HIPEREOSINOFÍLICA}

'Oncology and Hematology Program, Instituto Israelita de Ensino e Pesquisa Albert Einstein, São Paulo SP, Brazil; ${ }^{2}$ Neurology Program, Instituto Israelita de Ensino e Pesquisa Albert Einstein, São Paulo SP, Brazil.

Received 18 November 2008, received in final form 12 March 2009. Accepted 6 May 2009.

Dr. Nelson Hamerschlak - Centro de Pesquisa Clínica / Instituto Israelita de Ensino e Pesquisa Albert Einstein - Avenida Albert Einstein 627 / 701 05651-901 São Paulo SP - Brasil. E-mail: hamer@einstein.br 

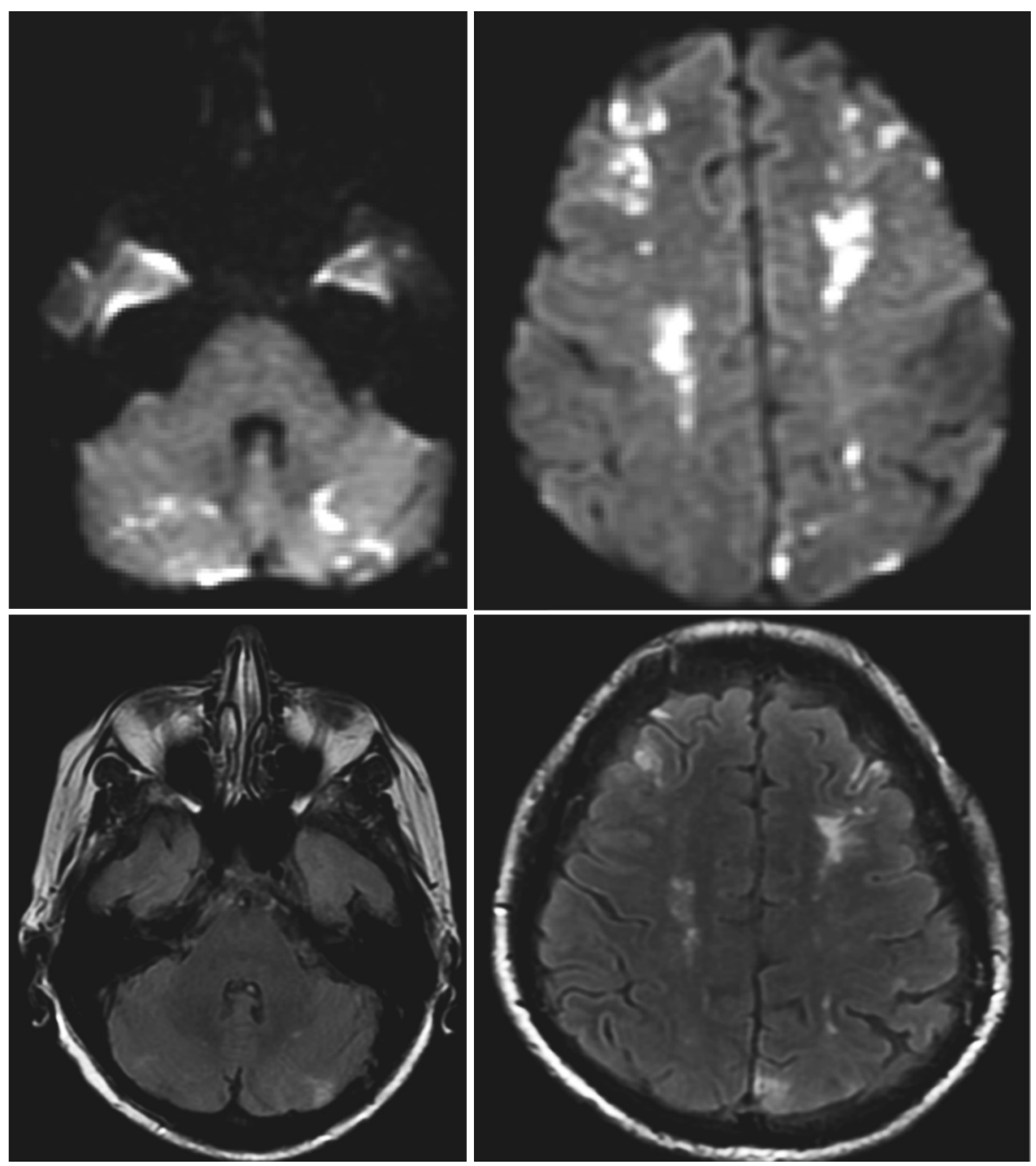

Figure. Magnetic resonance imaging of a 63-year old woman showing axial diffusion and fluid-attenuated inversion recovery (FLAIR) weighed images of the posterior fossa and cerebral hemispheres showing multiple small foci of diffusion restriction and high signal in FLAIR, consistent with acute infarctions in watershed distribution.

ly and after that, oral anticoagulation with warfarin (maintaining international normalized ratio, INR, of 2.0-3.0), as well as cytotoxic therapy (hydroxyurea, $1 \mathrm{~g} /$ day, interferon alfa, 5,000,000 units/day, and oral prednisone, $40 \mathrm{mg} /$ day). She was discharged with significant improvement of her neurological symptoms and her eosinophils count. According to her hematological response and the absence of an embolic source, she was discharged with an oral antiplatelet agent, clopidogrel, $75 \mathrm{mg}$ once a day.

One week later, the patient was readmitted with significant neurological impairment with cerebellar ataxia and urinary incontinence. Despite oral antiplatelet therapy with $100 \mathrm{mg}$ of aspirin and significantly lower eosinophil count $(6,480$ eosinophils $/ \mathrm{mm}^{3}$ ), brain MRI disclosed new areas of infarction.

She was submitted to a new investigation of the ischemia, and no embolic source was found, even at transcranial Doppler, performed again. She was treated with anticoagulants agents once more, despite these results, and no worsening in neurologi- cal functions was noticed. She is now under neurological rehabilitation, with special attention to gait and cognitive impairments.

\section{DISCUSSION}

Neurological impairment due to thromboemboli in HES usually occurs throughout the course of HES in conjunction with cardiac involvement ${ }^{7}$. In 52 patients with HES, cerebrovascular thromboembolic disease affected six patients and was associated with heart involvement in five ${ }^{6}$. In our patient, a complete workup was performed to exclude thromboemboli as cause for the cerebrovascular disease. However, despite oral antiplatelet therapy, the patient presented with worsening of neurological function.

Leukostasis is not cited in the literature as a possible complication of high eosinophil count in hypereosinophilic syndrome. In our case, there were no signs or laboratory findings suggesting leukostasis. In fact, despite 
control of eosinophil count, new neurological impairment was observed.

Recently, few reports of watershed infarction in patients with HES have been published, suggesting a possible toxic direct effect of eosinophils ${ }^{7,8}$. Activated eosinophils and their granular proteins including eosinophil cationin protein, major basic protein, are thought to modify coagulation and fibrinolysis in eosinophilia, resulting in thrombosis ${ }^{9}$. In fact, thrombosis in unusual sites in patients with HES has been described ${ }^{10}$. There is no consensus about the ideal management of thrombosis in such cases.

Patients with HES are usually diagnosed as having a worse prognosis when their eosinophils count is above $90,000 / \mathrm{ml}$. The case described here shows that patients with hypereosinophilic syndrome can have their eosinophils count increased very quickly and can present with recurrent ischemic events even when the eosinophil count is low. Watershed ischemia is a possible presentation of neurological involvement in HES, and there is no consent of the ideal management of such cases.

Today, with the development of new treatments as anti-interleukin-5, imatinib, alemtuzumab and other tyrosinokinase inhibitors, patients that are resistant to corticosteroid therapy should be submitted to other treatment modalities.

\section{REFERENCES}

1. Haferlach T, Bacher U, Kern W, Schnittger S, Haferlach C. The diagnosis of $\mathrm{BCR} / \mathrm{ABL}$-negative chronic myeloproliferative diseases (CMPD): a comprehensive approach based on morphology, cytogenetics, and molecular markers. Ann Hematol 2008;87:1-10.

2. Weller PF, Bubley GJ. The idiopathic hypereosinophilic syndrome. Blood 1994;83:2759-2779.

3. Tefferi A, Patnaik MM, Pardanani A. Eosinophilia: secondary, clonal and idiopathic. Br J Haematol 2006;133:468-492.

4. Gotlib J, Cross NC, Gilliland DG. Eosinophilic disorders: molecular pathogenesis, new classification, and modern therapy. Best Pract Res Clin Haematol 2006;19:535-569.

5. Tefferi A, Vardiman JW. Classification and diagnosis of myeloproliferative neoplasms: the 2008 World Health Organization criteria and point-of-care diagnostic algorithms. Leukemia 2008;22:14-22.

6. Moore PM, Harley JB, Fauci AS. Neurologic dysfunction in the idiopathic hypereosinophilic syndrome. Ann Intern Med 1985;102:109-114.

7. Sarazin M, Caumes E, Cohen A, Amarenco P. Multiple microembolic borderzone brain infarctions and endomyocardial fibrosis in idiopathic hypereosinophilic syndrome and in Schistosoma mansoni infestation. J Neurol Neurosurg Psychiatry 2004;75:305-307.

8. McMillan HJ, Johnston DL, Doja A. Watershed infarction due to acute hypereosinophilia. Neurology 2008;70:80-82.

9. Sakuta R, Tomita Y, Ohashi M, Nagai T, Murakami N. Idiopathic hypereosinophilic syndrome complicated by central sinovenous thrombosis. Brain Dev 2007;29:182-184.

10. Kojima K, Sasaki T. Veno-occlusive disease in hypereosinophilic syndrome. Intern Med 1995;34:1194-1197. 